\title{
Infection with Influenza Virus Induces IL-33 in Murine Lungs
}

\author{
Ronan Le Goffic ${ }^{1}$, Muhammad Imran Arshad², Michel Rauch², Annie L'Helgoualc'h², \\ Bernard Delmas', Claire Piquet-Pellorce ${ }^{2}$, and Michel Samson ${ }^{2}$ \\ ${ }^{1}$ Unité de Virologie et Immunologie Moléculaires, Unité de Recherche 892, Institut National de la Recherche Agronomique, Jouy-en-Josas, France; \\ and ${ }^{2}$ Equipe Accueil 4427 Signalisation et Réponses aux Agents Infectieux et Chimiques, Université de Rennes 1, Institut Fédératif de Recherche 140, \\ Rennes, France
}

\begin{abstract}
IL-33, a novel IL-1 family member, is crucially expressed and involved in pulmonary diseases, but its regulation in viral diseases such as influenza $A$ virus (IAV) remains unclear. This study aimed to characterize the expression and release of IL-33 in lungs of IAVinfected mice in vivo and in murine respiratory epithelial cells (MLE15) in vitro. Our results provide evidence of up-regulation of IL-33 mRNA in IAV-infected murine lungs, compared with noninfected control mice. The overexpression of IL-33 was positively correlated with a significant increase in mRNA encoding the proinflammatory cytokines TNF- $\alpha$, IFN- $\gamma$, IL-1 $\beta$, and IL- 6 , and was also associated with an increase in IFN- $\beta$ mRNA. A profound overexpression of IL-33 protein was evident in IAV-infected murine lungs and bronchoalveolar lavages of influenza-infected mice, compared with low concentrations in naive lungs in vivo. Immunolocalization highlighted the cellular expression of IL-33 in alveolar epithelial and endothelial cells, along with increased infiltrate cells in virusinfected lungs. Further in vitro experiments showed an induction of IL-33 transcript-in MLE-15 cells and human epithelial cells (A549) infected with different strains of IAV in comparison with noninfected cells. In conclusion, our findings evidenced a profound expression of IL-33 in lungs during both in vivo and in vitro IAV infections, suggesting a role for IL-33 in virus-induced lung infections.
\end{abstract}

Keywords: IL-33; influenza virus; pulmonary infection

IL-33 is a novel member of the IL-1 cytokine family, sharing structural homology with cytokines of the IL-1 family such as IL-1 $\alpha$, IL-1 $\beta$, and IL-18 (1). IL-33 presents dual functionality. First, it is localized in the nucleus (as a chromatin-associated factor), and functions as a transcriptional repressor (2). However, its exact biological functions remain unknown. Second, in terms of cytokine function, IL-33 induces its biological activity by the interaction of a heterodimer composed of IL-1 receptorlike 1 (ST1) and IL-1 receptor accessory protein receptors. ST2 is present in both transmembrane (ST2L) and secreted (sST2) forms, and its transmembrane form is expressed primarily in Th2 cells and mast cells (3). Soluble IL-33 is known to increase the secretion of Th2 cytokines (IL-4, IL-5, and IL-13) (1) and to act as a chemoattractant for Th2 lymphocytes both in vivo and in vitro, elucidating its ability to induce a Th2 immune response

(Received in original form December 22, 2010 and in final form May 27, 2011)

This work was supported by Institut National de la Santé Et de la Recherche Médicale, the Institut National de la Recherche Agronomique, the Ministère de I'Education Nationale de la Recherche et de la Technologie, Région Bretagne, the Ligue contre le Cancer, and Institut Fédératif de Recherche 140. M.I.A. was supported by a doctoral fellowship from the Government of Pakistan (via the Higher Education Commission, University of Agriculture, Faisalabad, Pakistan).

Correspondence and requests for reprints should be addressed to Michel Samson, Ph.D., EA 4427 SeRAIC, Université de Rennes 1, 2 Avenue du Prof. Léon Bernard, 35043 Rennes Cedex, France. E-mail: michel.samson@univ-rennes1.fr

Am J Respir Cell Mol Biol Vol 45. pp 1125-1132, 2011

Originally Published in Press as DOI: 10.1165/rcmb.2010-0516OC on June 3, 2011

Internet address: www.atsjournals.org in different inflammatory diseases (4). IL-33 is also reported to be a crucial amplifier of innate immune responses during allergic airway and autoimmune diseases (5).

Because of its wide expression in several tissues and cell types, IL-33 is involved in many immunopathologies such as arthritis (6), Crohn's disease (2), and anaphylactic shock (7), and autoimmune diseases such as systemic lupus erythematous (8), chronic hepatitis (9), and chronic pancreatitis (10). Regarding the cellular source of IL-33 in these pathologies, smooth muscle cells, fibroblasts, keratinocytes, dendritic cells, activated macrophages, and epithelial cells were reported to express IL-33 (9-11).

IL-33 is also reported to be involved in pulmonary disease. According to recent findings, the overexpression of IL-33, TNF- $\alpha$, and IFN- $\gamma$ is concomitant with severe asthma, whereas TNF- $\alpha$ and IFN- $\gamma$ exert synergic effects in the regulation of IL-33 expression in airway smooth muscle cells (12). In allergic airway inflammation, the role of IL-33 may involve hastening the pathology as in asthma, where blocking the IL-33/ST2 pathway is favorable in limiting disease severity (13). More recently, the increased expression of IL-33 and other inflammatory mediators such as IL-5, IL-8, IL-13, and IgE were associated with spontaneous pulmonary inflammation in murine IL-33 (mIL-33) transgenic mice (14).

The induction of Th2-cytokine-associated lung inflammation was observed in mice after the inhalation of respiratory syncytial virus (RSV), which was abrogated by anti-ST2 monoclonal antibody $(\mathrm{mAb})$ treatment, suggesting a role of ST2 inhibition in virally induced pulmonary infection (15). To date, however, few data are available regarding the role of IL-33 in viral diseases, and particularly in pulmonary infectious diseases such as influenza A virus (IAV).

IAV, the causative agent of flu, is a worldwide health problem. Although vaccine provides good protection against epidemic flu strains, recent outbreaks highlight the need for a better understanding of the mechanisms behind IAV-induced disease. Indeed, in 2009, a new strain of IAV appeared and spread worldwide, causing the first flu pandemic of the 21 st century (16). IAV can cause mild to severe respiratory illness, and can lead to death. Acute pneumonia is an important complication of IAV. This kind of complication is mainly attributable to highly pathogenic strains, such as H5N1. Highly pathogenic strains trigger severe damage to the lungs, and consequently tremendous amounts of proinflammatory cytokines and chemokines are produced by influenzainfected cells. This uncontrolled inflammatory cascade is called "cytokine storm," and often leads to death (17).

Therefore, to provide new insight into the induction of cytokines during infection with IAV, we studied IL-33 in H1N1 IAVinduced pulmonary infection in mice and the induction of IL-33 in response to infections with different strains of IAV in cultured human and murine epithelial cells. Our findings provide evidence of the profound expression of IL-33 in virus-infected lung tissue in correlation with other proinflammatory cytokines, suggesting a role for IL-33 in virally induced respiratory pathologies. 


\section{MATERIALS AND METHODS}

\section{Viral and Murine Strains}

Influenza A/WSN/1933 (H1N1) was used for in vivo and in vitro experiments. Influenza virus strains PR8 (influenza A/Puerto Rico/8/1934; H1N1), Udorn (influenza A/Udorn/307/1972; H3N2), and Mass65 (influenza A/Turkey/Massachusetts/3740/1965; H6N2) were also tested in vitro during the infection of murine and human epithelial cell lines. Virus stocks were produced as previously described (18). Female C57Bl/6 mice, aged 8 weeks, were purchased from the Centre d'Elevage R. Janvier (Le Genest Saint Isle, France), were bred in specific pathogen-free conditions, and were used after 2 weeks of adaptation in our rodent facility. For experiments involving infection with IAV, mice were housed in ventilated cages under negative pressure with high efficiency particulate air-filtered air. Mice were treated in accordance with the guidelines of the Institut National de la Recherche Agronomique, in compliance with European animal welfare regulations.

\section{Infection of Animals and Collection of Bronchoalveolar Lavage Fluid}

The murine lethal dose $50\left(\mathrm{MLD}_{50}\right)$ of viral stock used in this study was determined to be $1.5 \times 10^{5}$ plaque-forming units (PFU) per mouse. Anesthetized mice were infected intranasally with $50 \mu \mathrm{l}$ of PBS containing $1.5 \times 10^{5} \mathrm{PFU}\left(1 \mathrm{MLD}_{50}\right)$ or $1 \times 10^{6} \mathrm{PFU}\left(6.5 \mathrm{MLD}_{50}\right.$, i.e., the lethal dose) of IAV (three and eight mice per group, respectively, with four mice as control subjects, injected with PBS only).

Mice were observed for signs of morbidity and death for 12 days. Alternatively, mice were killed at different time points, and bronchoalveolar lavage (BAL) and lungs were collected as previously described (19).

\section{Detection of IL-33 at the mRNA and Protein Levels}

First-strand cDNA was synthesized from total RNA extracted from lung tissue, and real-time quantitative PCR (qPCR), using specific primer pairs (Table 1), was performed as previously described (9). The absolute mRNA copy number of IL-33 was determined in murine lungs (control versus infected) by qPCR, using a standard curve of murine IL-33 purified plasmid (a gift from Jean-Philippe Girard, Centre National de la Recherche Scientifique, Unité Mixte de Recherche 5089-Institut de Pharmacologie et de Biologie Structurale, Toulouse, France). The immunolocalization of IL-33 was performed on paraformaldehydefixed cryosections of murine lungs. Cryosections were permeabilized with $0.1 \%$ Triton X-100, and goat IgG anti-mouse IL-33 $(2 \mu \mathrm{g} / \mathrm{mL}$, R\&D Systems, Lille, France) and rat IgG anti-mouse CD45 $(2 \mu \mathrm{g} / \mathrm{mL}$, BD Biosciences, Le Pont de Claix, France) antibodies were used to identify IL-33-positive or CD45-positive cells. Further detection of fluorescence was performed with secondary bovine anti-goat IgG Cy5 and donkey anti-rat IgG $\mathrm{Cy} 3$ antibodies (Jackson Immunoresearch Laboratories, West Grove, PA), and 4',6-diamidino-2-phenylindole was used for counterstaining, as previously described (9). The expression of IL-33 was also evaluated by Western blot analysis of total protein extracted from lung tissues separated on a $16 \%$ polyacrylamide gel, using the polyclonal anti-mouse IL-33 antibody $(0.2 \mu \mathrm{g} / \mathrm{ml})$. Protein bands were detected by enhanced chemiluminescence, and MultiGauge software (Fujifilm, Tokyo, Japan) was used to measure the bands' intensity.

\section{Murine and Human Cell Culture}

Murine lung epithelial (MLE)-15 cells (Type II pneumocyte cell line) were grown as recommended by Wikenheiser and colleagues (20). For infection, cells were washed with FBS-free medium and incubated with virus at a multiplicity of infection (MOI) of 5, and then diluted in FCSfree medium. Human and murine lung epithelial cell lines (Type II pneumocytes; A549 and MLE-15, respectively) were infected with different strains of IAV (WSN, PR8, Udorn, and Mass65) along with a control for 24 hours at an MOI of 5, and the relative transcript level of murine or human IL-33 was measured. The observed cytopathic effects of IAV strains are indicated as "+" (cells appear vacuolated and granular; Mass65), “++" (many round, swollen epithelial cells are present; PR8), " +++ " (lysis of cell layer, 20\%; WSN), and " $++++"$ (important lysis of the cell layer, $>50 \%$; Udorn).

\section{Measurement of Cytokines}

Murine IL-33, IL-6, TNF- $\alpha$, and IFN- $\gamma$ concentrations in BAL were determined using DuoSet ELISA kits obtained from R\&D Systems.

\section{Statistical Analysis}

The results in each model are represented as the mean \pm SD of each group, and are representative of three independent experiments. The Student $t$ test was used for comparisons of control group parameters with those of other treatment groups. Correlations between continuous variables were analyzed by using GraphPad Prism5 software (GraphPad Software, San Diego, CA).

\section{RESULTS}

\section{Induction of IL-33 mRNA in Lungs of IAV-Infected Mice Is Consistent with the Induction of Proinflammatory Cytokines}

We investigated whether IL-33 transcripts are up-regulated in viral (influenza A/WSN/1933; H1N1) pulmonary infection. In our first attempt, we assessed the expression of IL-33 in the lungs of mice infected with a high dose of IAV $\left(10^{6} \mathrm{PFU} /\right.$ mouse $), 3$ days after infection. As expected, infected mice exhibited significant weight loss, compared with noninfected control mice (Figure 1A). We found a significant $(P<0.001)$ increase in the relative mRNA expression of IL-33 in the virus-infected group, compared with noninfected control mice (Figure 1B). Because IL-33 is known to be expressed constitutively in lung tissue, we measured the absolute mRNA copy number of IL-33 in infected and control mice, and we observed a significant $(P<0.001)$ increase (4.3-fold) in the expression of IL-33 in infected mice compared with noninfected mice (Figure 1B). A similar increase in the mRNA expression of IFN- $\beta$ was evident in virally

TABLE 1. SEQUENCE OF PRIMERS FOR REAL-TIME RT-PCR

\begin{tabular}{lll}
\hline Gene & \multicolumn{1}{c}{ Forward } & \multicolumn{1}{c}{ Reverse } \\
\hline IAV & 5'AAGACCAATCCTGTCACCTCTGA3' & 5'CAAAGCGTCTACGCTGCAGTCC3' \\
Murine $\beta$-actin & 5'TGTTACCAACTGGGACGACA3' & 5'GGGGTGTTGAGGTCTCAAA3' \\
Murine $18 S$ & 5'CGCCGCTAGAGGTGAAATTC3' & 5'TTGGCAAATGCTTTCGCTC3' \\
Murine IFN- $\beta$ & 5'CCCTATGGAGATGACGGAGA3' & 5'CTGTCTGCTGGTGGAGTTCA3' \\
Murine IL-33 & 5'ATGGGAAGAAGCTGATGGTG3' & 5'CCGAGGACTTTTGTGAAGG3' \\
Murine IL-1 $\beta$ & 5'GAAGAAGTGCCCATCCTCTG3' & 5'AGCTCATATGGGTCCGACAG3' \\
Murine TNF- $\alpha$ & 5'TAGCTCCCAGAAAAGCAAGC3' & 5'TTTCTGGAGGGAGATGTGG3' \\
Murine IFN- $\gamma$ & 5'AGGTCAACAACCCACAGGTC3' & 5'ATCAGCAGCGACTCCTTTC3' \\
Murine IL-6 & 5'CCGGAGAGGAGACTTCACAG3' & 5'CAGAATTGCCATTGCACAAC3' \\
Human IL-33 & 5'ACACTCCAGGATCAGTCTTG3' \\
Human $\beta-$-actin & 5'AATCAGGTGACGGTGTTG3' & 5'CTCCTTAATGTCACGCACGA3' \\
\hline
\end{tabular}

Definition of abbreviation: IAV, influenza A virus. 
A B
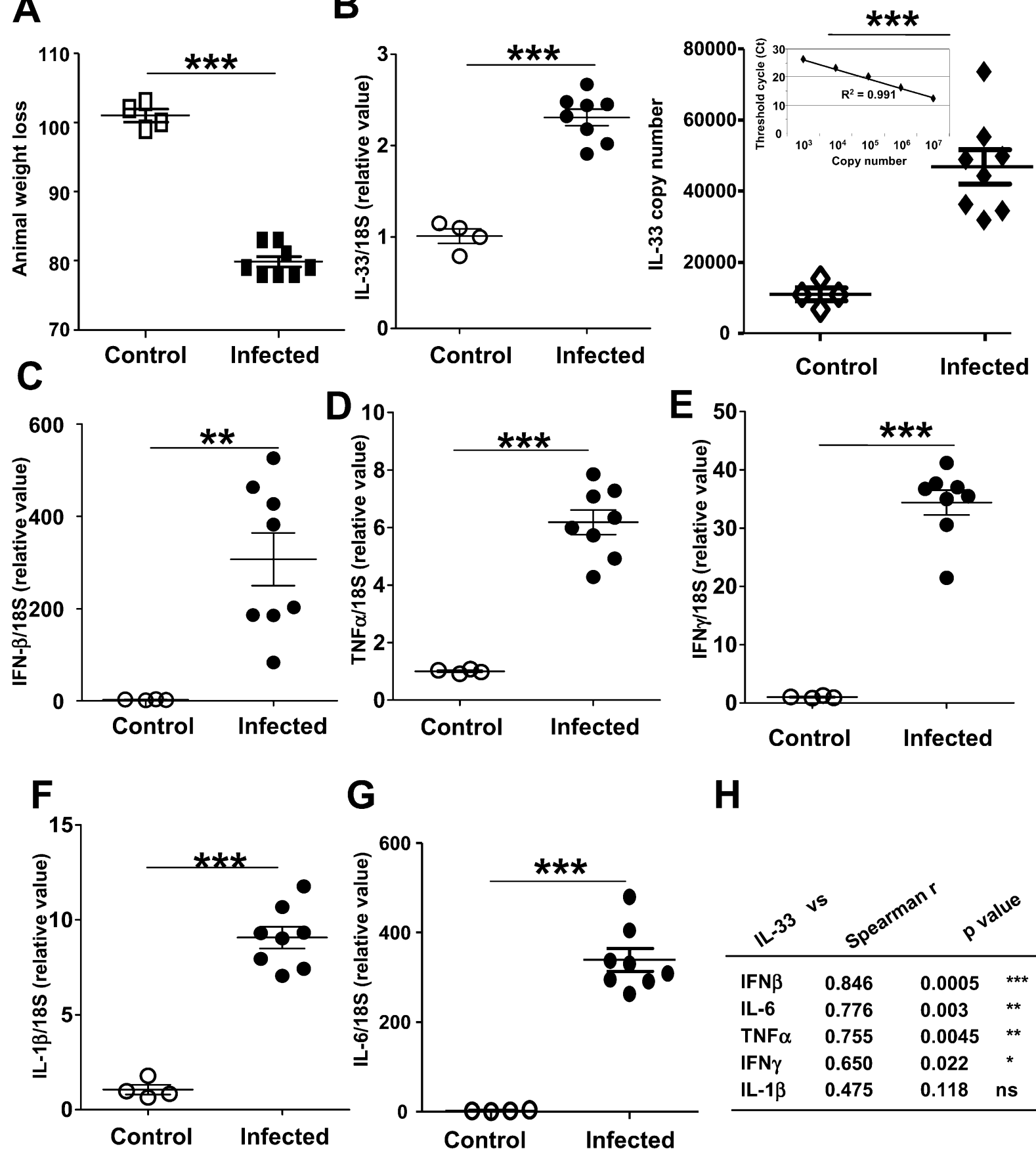

H

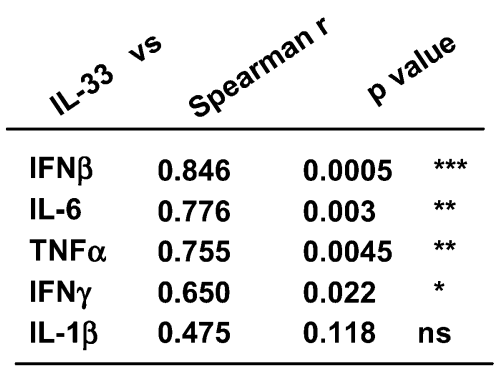

Figure 1. Measurement of animal weight loss and mRNA expression of IL-33, IFN- $\beta$, TNF $\alpha$, IFN- $\gamma$, IL-1 $\beta$, and IL- 6 in lungs of mice. Mice (C57BI/6) were infected intranasally with $10^{6}$ plaque-forming units (PFU) of the influenza A virus (IAV) strain (H1N1), and killed after 3 days of infection. ( $A$ ) Percentages of initial weight comparisons between infected and noninfected mice on Day 3 after infection. Measurement of mRNA expression of IL-33 (B), IFN- $\beta(C)$, TNF- $\alpha(D)$, IFN- $\gamma(E)$, IL-1 $\beta(F)$, and IL-6 $(G)$ in lungs according to quantitative RT-PCR. The expression of mRNA is represented as a relative value of infected mice $(n=8)$ compared with noninfected control mice $(n=4)(B-G)$, as well as an absolute IL-33 mRNA copy number $(B)$. $(H)$ Correlation of the mRNA induction of IL-33 with mRNA concentrations of TNF- $\alpha$, IFN- $\beta$, IFN- $\gamma$, IL- $1 \beta$, and IL- 6 in lungs of mice on Day 3 . In all graphs, the value for each mouse is plotted, and the line indicates the mean ( \pm SD) value of each group, with significance according to the Student $t$ test ${ }^{* *} P<0.01$ and $\left.{ }^{* * *} P<0.001\right)$.

infected mice $(P=0.002)$ compared with the control group (Figure $1 \mathrm{C})$. The induction of the IL-33 transcript was significantly and positively correlated with IFN- $\beta$ transcript levels (Spearman $r=0.846, P=0.0005)$ (Figure $1 \mathrm{H})$, and was negatively correlated with loss of weight (Spearman $r=-0.700, P=0.011$ ). These results suggest an association between the onset of viral infection and the expression of IL-33 during virally induced lung infection.
Because previous studies reported that the proinflammatory cytokines TNF- $\alpha$, IFN- $\gamma$, IL-1 $\beta$, and IL- 6 induced IL-33 in hepatitis or pancreatitis $(9,21)$, we assessed the mRNA expression of these cytokines on Day 3 after infection with a high dose of virus, that is, $10^{6} \mathrm{PFU} / \mathrm{mouse}$, to characterize further the potential role of TNF- $\alpha$, IFN- $\gamma$, IL- $1 \beta$, and IL- 6 in virus-infected mice. We found a significant increase $(P<0.05)$ in the mRNA expression 
A

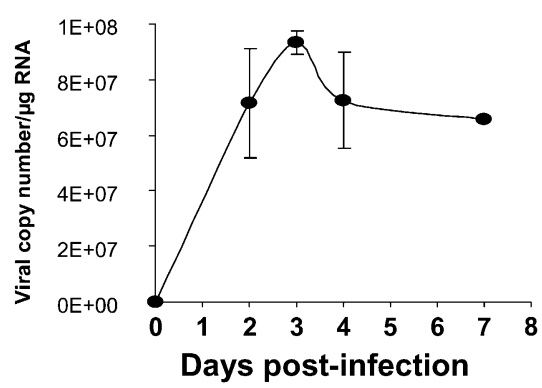

C

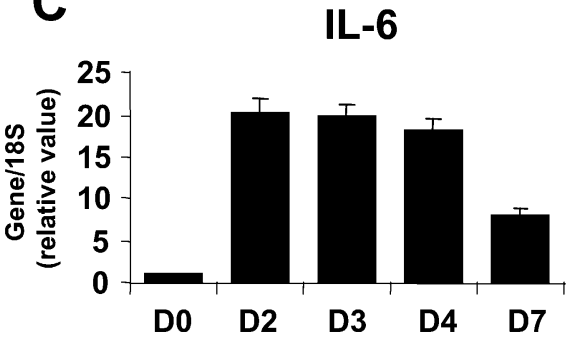

$\mathbf{E}$

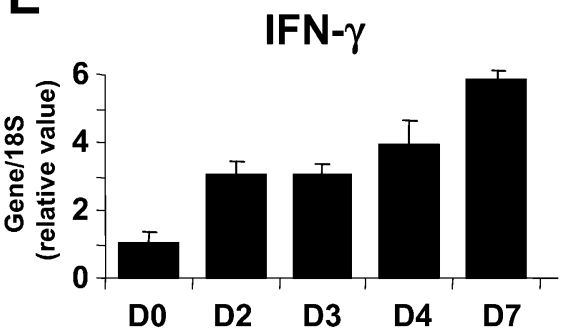

\section{B}

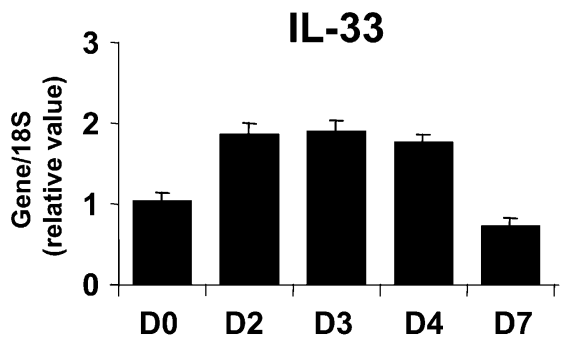

Figure 2. Kinetics of mRNA expression of IAV, IL-33, IL-6, TNF $\alpha$, IFN- $\gamma$, and IL-1 $\beta$ in lungs of mice infected with a moderate dose of IAV. Mice $(\mathrm{C} 57 \mathrm{BI} / 6)$ were killed on Day 0 or $2-7$ days (D2, D3, D4, and D7) after infection with $1.5 \times 10^{5}$ PFU of IAV per mouse. Measures of mRNA expression of IAV $(A), \mathrm{IL}-33(B), \mathrm{IL}-6(C), \mathrm{IL}-1 \beta(D)$, IFN- $\gamma(E)$, and TNF- $\alpha(F)$ in lung tissue by quantitative RT-PCR are represented as relative values compared with noninfected mice (means \pm SD; $n=3)$.

of TNF- $\alpha$, IFN- $\gamma$, IL-1 $\beta$, and IL- 6 in infected mice compared with control mice (Figures 1D-1G). A significant correlation was evident between the mRNA induction of IL-33 with mRNA levels of TNF- $\alpha$, IFN- $\gamma$, and IL-6, but not with IL-1 $\beta$ (Figure $1 \mathrm{H}$ ).

Kinetics of mRNA Expression of IL-33, IL-6, TNF- $\alpha$, IFN- $\gamma$, and IL-1 $\beta$ with a Moderate Dose of Influenza Virus Lung Infection

We further investigated the expression of proinflammatory cytokines, including TNF- $\alpha$, IFN- $\gamma$, IL-1 $\beta$, IL-6, and IL-33, with a moderate dose of virus $\left(1.5 \times 10^{5} \mathrm{PFU} / \mathrm{mouse}\right.$, i.e., $\left.1 \mathrm{MLD}_{50}\right)$, from Day 2 up to Day 7 after infection. We also followed the viral copy number during lung infection (from Day 0 to Day 7). Our results indicated a high viral copy number on Day 2, a peak on Day 3, and a raised viral copy number on Days 4-7 (Figure 2A). The mRNA expression of IL-33 (Figure 2B), IL-6 (Figure 2C), IL-1 $\beta$ (Figure 2D), IFN- $\gamma$ (Figure 2E), and TNF- $\alpha$ (Figure 2F) presented a similar profile, with increased expression on Day 2, a peak on Day 3, and maintained expression on Days 4-7. The mRNA expression of IFN- $\gamma$ increases slowly, with maximum mRNA expression on Day 7 (Figure 2E). These kinetics of cytokine responses clearly evidenced IL-33 as a member of the classic proinflammatory cytokines, namely, IL- 6 , IFN- $\gamma$, TNF- $\alpha$, and IL-1 $\beta$, during the acute phase of viral lung infection.

\section{Expression of IL-33 in Bronchoalveolar Endothelial and Epithelial Cells during IAV Lung Infection}

To investigate the expression of IL-33 during virus-induced pulmonary infection, we performed histologic and co-immunolocalization studies. As expected, lung tissue revealed normal lung structure in noninfected mice (Figure 3A), whereas IAV-infected mice manifested the destruction of alveolar structure and accumulation of infiltrate cells in the lumen of bronchi in virus-infected lungs (Figure $3 \mathrm{~B})$. At the cellular level, IL-33 was expressed in alveolar epithelial and endothelial cells in naive lungs (Figures $3 \mathrm{~B}$ and $3 \mathrm{E}$, green) and in virus-infected lungs (Figures 3D and 3F). However, the infiltrate cells (pan-leukocytes, CD $45^{+}$) were strongly increased in the lumen of bronchi of influenza-infected lungs (Figures $3 \mathrm{D}$ and $3 \mathrm{~F}$, pink), compared with naive lungs, as a consequence of viral infection.

\section{IL-33 Protein Is Up-Regulated during IAV Pulmonary Infection of Mice}

We also evaluated the level of protein expression of IL-33 in virus-infected lungs versus naive lungs. The Western blot analysis of total proteins extracted from lung tissue indicated an increased protein expression of IL-33, with 32-35-kD molecular weights (active form) in virus-infected lungs (Figure 4A). Ratios of signal densities of IL-33/ $\beta$-actin clearly showed an increased expression on Day 3 in post-infected lungs compared with noninfected lungs (Figure 4B). These findings provide evidence that the IL-33 observed in lungs is active and highly inducible during pulmonary viral infections.

\section{Increased Expression of Soluble Protein of IL-33 in BAL of IAV-Infected Mice}

To investigate the presence of IL-33 in BAL fluid, we measured the protein expression of IL-33 by ELISA in both control and 
Non-infected
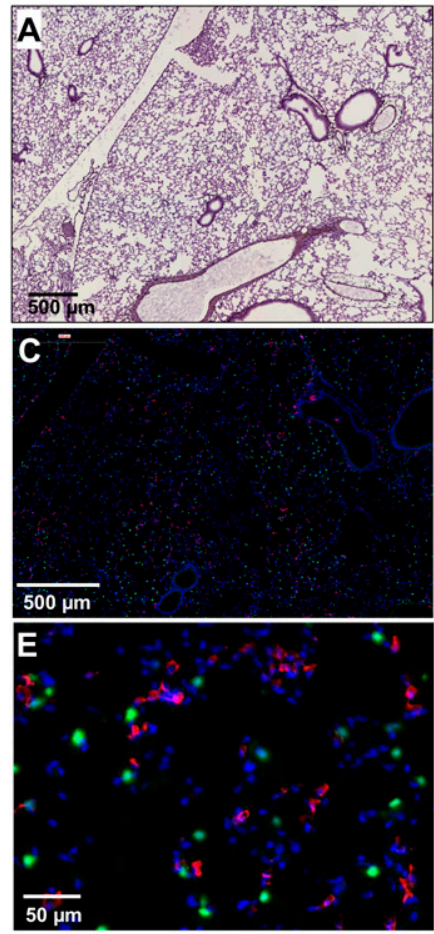

Figure 3. Co-immunolocalization of murine IL-33 (mIL-33) and CD45 in lungs of mice infected with IAV and naive murine lungs on Day 3 after infection. Mice were infected with a high dose of IAV $\left(10^{6} \mathrm{PFU} /\right.$ mouse $)$ and were killed 3 days after infection. Lungs from noninfected $(A, C$, and $E)$ and infected $(B, D$, and $F)$ mice were collected and preserved in $4 \%$ formalin to perform hematoxylin-eosin staining and the immunolocalization of IL-33 (green) and CD45 (pink). Nuclei were stained with 4',6-diamidino-2phenylindole (blue). Scale bars $=50 \mu \mathrm{m}$ or $500 \mu \mathrm{m}$, as indicated.

virus-infected mice. We found a strong and significant $(P=0.009)$ increase in the protein expression of IL-33 in the BAL fluid of virus-infected mice compared with the control group (Figure 5).

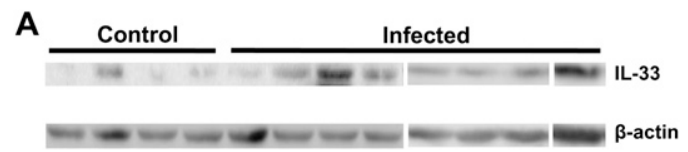

B

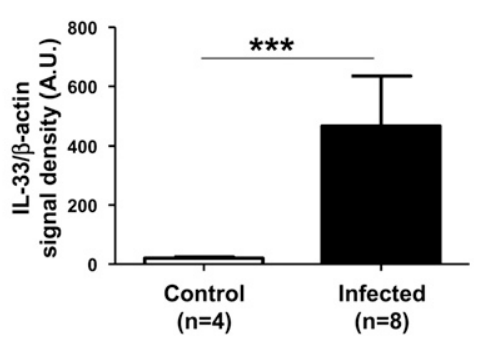

Figure 4. Western blot analysis of IL-33 protein expression in lungs of mice infected or not infected with IAV. Mice were infected with a high dose $\left(10^{6}\right.$ $\mathrm{PFU} /$ mouse) of IAV and were killed on Day 3 after infection to harvest their lungs. $(A)$ Total protein extracts of lung tissues from either control $(n=4)$ or infected mice $(n=8)$ were analyzed by Western blotting, using antimlL-33 antibody and anti- $\beta$-actin antibody as housekeeping protein. (B) Signal densities of IL-33 and $\beta$-actin in virus-infected and control murine lungs were measured by MultiGauge software for image analysis. The ratios of signal densities of IL-33/ $\beta$-actin are represented as means \pm SD of each group ( ${ }^{\star *} P<0.01$, Student $t$ test). A.U. = arbitrary unit.

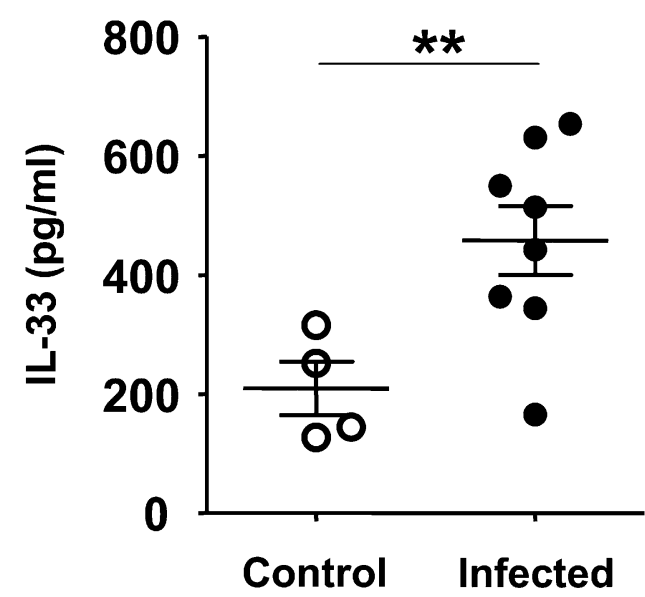

Figure 5. Quantification of soluble IL-33 protein in bronchoalveolar lavage (BAL) of mice infected with influenza virus. BAL fluids were collected from mice infected $(n=8)$ or not infected (control, $n=4)$ with a high dose $\left(10^{6} \mathrm{PFU} / \mathrm{mouse}\right)$ of IAV, 3 days after infection. The value for each mouse is plotted, and the line indicates the mean $( \pm S D)$ value of each group, with significance according to Student $t$ test ( $\left.{ }^{* \star} P<0.01\right)$.

In addition, we found a significant correlation $(P=0.026, r=$ 0.636 ) between the mRNA expression of IL-33 in the lungs and the protein expression of IL-33 in the BAL fluid of the same mice. These findings further suggest that IL-33 is induced and secreted during the early phase of IAV lung infection.

\section{Increased Expression of Soluble Protein of IL-33 in IAV-Infected Lung Epithelial Cells In Vitro}

We investigated the release of IL-33 in a conditioned medium of lung epithelial cells infected by IAV in vitro. We used the transformed murine respiratory epithelial cell line, MLE-15, which has many structural and biological features of alveolar Type II cells. We found a threefold increase $(P<0.001)$ of IL-33 mRNA expression in IAV-infected MLE-15 cells compared with noninfected cells (Figure 6A). As expected, during such culture conditions with the virus, the mRNA expression of IFN$\beta$ was significantly augmented $(P<0.001)$ in IAV-infected MLE-15 cells compared with noninfected cells (Figure 6B). The kinetics of the mRNA levels of IL-33 in IAV-infected MLE-15 cells revealed an increasing trend at 20 hours after infection, reaching maximum levels at 40 hours after infection (Figure 6C). Similarly, soluble IL-33 protein in a conditioned medium of IAV-infected MLE-15 cells demonstrated a rising trend at 20 hours after infection, with a peak of protein secretion at 40 hours after infection (Figure 6D). Such a release of IL-33 appears to be concomitant with the lysis of IAVinfected cells (Figure 6E). We further examined the effects of the infection of MLE-15 cells, using different strains (PR8, H1N1; Udorn, H3N2; and Mass65, H6N2) of IAV, and measured the mRNA expression of IL-33 accordingly. The PR8 and Mass65 strains significantly induced the mRNA expression of IL-33 at a moderate level, as with the WSN strain. However, the Udorn strain induced more elevated (11.6-fold, $P<0.001)$ transcript levels of IL-33 compared with control samples, which may be attributable to the increased cytopathic effect of the Udorn strain in vitro (Figure $6 \mathrm{~F}$ ). These in vitro results further confirm our in vivo findings of the induction of IL-33 in bronchial epithelial cells and its release in BAL during IAV infection, suggesting a role for IL-33 as an early cytokine mediator during viral infection. 
A

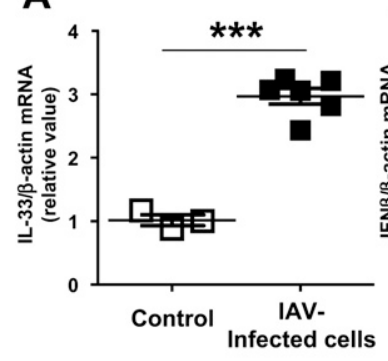

D

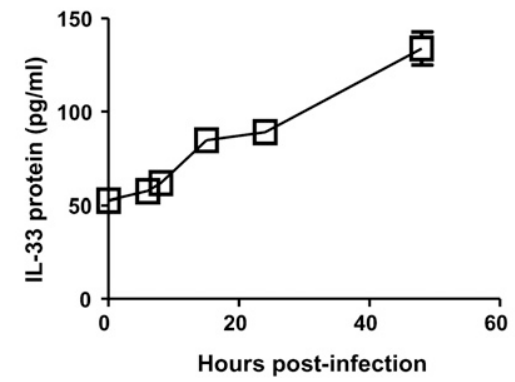

F

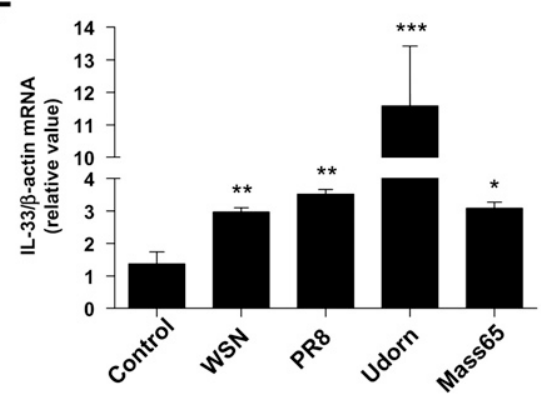

C

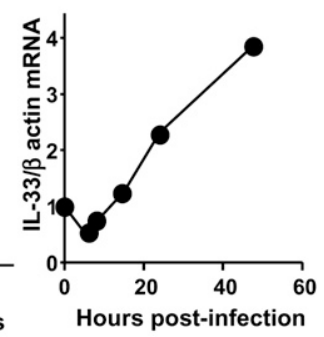

E

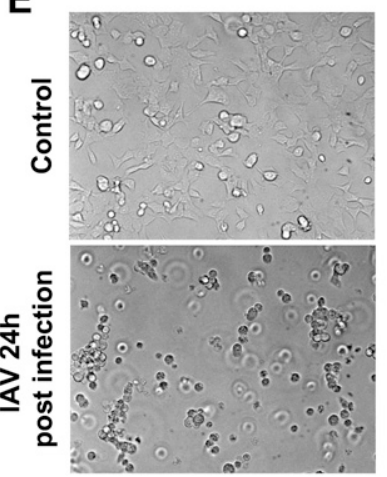

Figure 6. Expression of IL-33 in murine respiratory epithelial (MLE-15) cells infected with IAV. MLE-15 cells were infected or not infected with IAV at a multiplicity of infection (MOI) of 5 . After 24 hours of infection, the expressions of IL-33 (A) and IFN- $\beta(B)$ mRNA were measured by quantitative RT-PCR. The results were normalized with the level of $\beta$-actin gene expression, and presented as relative value of infected cells $(n=6)$ compared with noninfected cells $(n=3)$. The kinetics of IL-33 mRNA expression (C) and IL-33 protein secretion as quantified by ELISA in a conditioned medium $(D)$ of infected MLE-15 cells are shown. (E) Representative photographs of MLE-15 cell culture after 24 hours of infection with IAV. MLE-15 cells were infected with different strains of IAV, namely, WSN (H1N1), Puerto Rico 8 (PR8; H1N1), Udorn (H3N2), and Massachusetts 65 (Mass65; H6N2), along with control samples at an $\mathrm{MOI}$ of 5 . ( $F$ ) After 24 hours of infection, the relative mRNA expression of $\mathrm{mlL}-33 / \beta$-actin was measured by quantitative RT-PCR. Data are expressed as mean values ( $\pm \mathrm{SD})$, with significance according to Student $t$ test $\left({ }^{*} P<0.05,{ }^{* *} P<0.01\right.$, and $\left.{ }^{* * *} P<0.001\right)$.

\section{Increased Expression of IL-33 in a Human Lung Epithelial Cell Line during Infection with IAV}

Finally, in terms of relevance to human disease, we infected the human pulmonary epithelial cell line A549 (Type II pneumocyte cell line) with different strains of influenza virus (WSN, PR8, Udorn, and Mass65), and measured the mRNA expression of IL-33 along with control samples. The WSN, PR8, and Mass65 strains induced a significant increase ( $\sim 2-4$-fold $)$ of IL-33 at transcript levels (Figure 7). However, the Udorn strain induced a more profound (8-fold) increase in IL-33 mRNA expression in human epithelial cell lines compared with control samples (Figure 7). These data strengthen the relevance of the induction of IL-33 in human pulmonary cells during infection with IAV, and suggest a possible role for this cytokine in viral pulmonary infections.

\section{DISCUSSION}

IL-33, a novel IL-1 family member (also named IL-1F11), is notably expressed and involved in lung pathologies such as asthma (12), allergic airway inflammation (13), and acute pulmonary inflammation (14). However, the expression and role of IL-33 during infectious lung diseases have been little studied, especially during pulmonary infection with IAV. The aim of the present study was to characterize the expression and release of IL-33 in the lungs of IAV-infected mice.

The expression of IL-33 in lung tissue appears both constitutive and inducible. Schmitz and colleagues (1) described high concentrations of murine IL-33 mRNA in naive lungs, along with the constitutive expression of IL-33 in smooth muscle cells and bronchial epithelial cells. Our results confirm the high expression of IL-33 at both the transcriptional (relative and absolute mRNA copy numbers: 11,000 IL-33 mRNA copies/20 ng total RNA) and protein (200 pg/ml in BAL) levels in lungs, and demonstrate IL-33-positive alveolar cells in noninfected mice. Moreover, our findings indicate that the expression of IL-33 is clearly induced in IAV-infected murine lungs, both at the mRNA (relative and absolute copy numbers) and protein levels. The overexpression of IL-33 at the protein level was observed via Western blotting in total protein extracts of IAVinfected murine lungs compared with naive lungs in vivo. This finding is further supported by in vitro findings, because a threefold induction of IL-33 transcript and protein release was observed in IAV-infected cultured lung epithelial cells compared with noninfected cells.

Compared with other cytokines, the secretion or release of IL-33 from cells may occur through a nonclassic secretion process. Indeed, IL-33 was proposed to play the role of an "alarmin" in the immune system during the death of necrotic cells, as does HMGB1. Moreover, IL-33 may be a "necrocrine," released passively from the nucleus or cytosol when cells are lysed (22). In lung pathologies, HMGB1 protein was recently reported to be released and overexpressed in the BAL of mice infected with RSV (23). Our results also demonstrated a robust release and up-regulated expression of IL-33 protein (2.2-fold increase) in the BAL of IAV-infected murine lungs. These data support the proposed role of IL-33 as an alarmin mediator, much like 


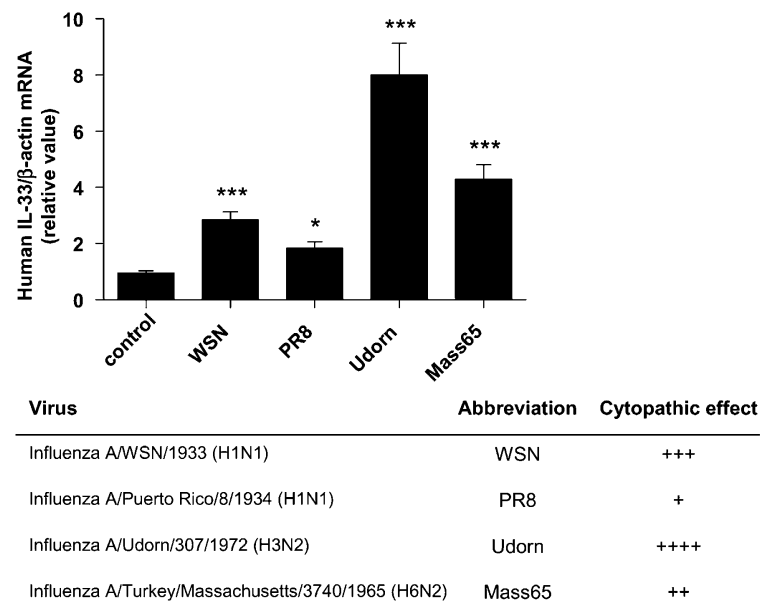

Figure 7. Relative mRNA expression of IL-33 in human respiratory epithelial cells (A549) infected with different strains of IAV. A549 cells were infected with different strains of IAV such as WSN, PR8, Udorn, and Mass65, along with control samples, at an MOI of 5 . After 24 hours of infection, human IL-33 mRNA expression was measured by quantitative RT-PCR. The results were normalized with the $\beta$-actin gene expression level and presented as relative values of infected cells $(n=3)$ compared with noninfected control cells $(n=4)$. Data are expressed as mean values ( $\pm \mathrm{SD})$, with significance according to Student $t$ test $\left({ }^{*} P<\right.$ $0.05,{ }^{* *} p<0.01$, and $\left.{ }^{* *} p<0.001\right)$.

HMGB1 during pulmonary viral infections. These results are in accordance with in vitro findings, where the amount of IL-33 protein in conditioned media of IAV-infected lung epithelial cells increased with the lysis of cultured cell layers by virus.

In all experiments, we detected the full-length IL-33 (32-35 kD) that represents the biologically active form. This finding suggests that, at this step of the infectious process, IL-33 is not cleaved and may act as a chemoattractant for lymphocytes, eosinophils, Th2 cells, and dendritic cells, as previously reported (4). Here we observed an important recruitment of infiltrate cells during infection with IAV, as shown by the CD45 panleukocyte marker. Among the functions that IL-33 can exert in lung tissue, recent studies demonstrated that cytokines of the IL- 1 family (IL- $1 \alpha$, IL-1 $\beta$, IL-18, and IL-33) play the role of an adjuvant for the induction of mucosal immunity (increased concentrations of $\operatorname{IgA}$ and $\mathrm{IgG}$ ) against IAV (24), suggesting a potent role for these cytokines, including IL-33, as co-enhancers of the immune response in viral disease. Furthermore, IAV, like $\mathrm{H} 1 \mathrm{~N} 1$, triggers severe damage to the lungs, and in consequence, large amounts of proinflammatory cytokines are released and aggravate the disease process (17). Our findings also demonstrated a significant increase of TNF- $\alpha$, IFN- $\gamma$, IL- $1 \beta$, and IL-6 in lungs of infected mice compared with control mice. A significant correlation was found between IL-33 and TNF- $\alpha$, IFN- $\gamma$, and IL-6, but not with IL-1 $\beta$. Moreover, concentrations of IL-33, IL-6, IL-1 $\beta$, IFN- $\gamma$, and TNF- $\alpha$ presented similar kinetics of the mRNA expression profile. After its release in the lungs after viral infection, IL-33 could play a role in the augmentation of disease severity. Suppressing its effects by the anti-ST2 mAb during RSV infection abrogated the lung pathology, providing evidence of a detrimental contribution of IL-33 to airway inflammation (15). Our findings coincide with recent studies that reported overexpressions of IL-33, TNF- $\alpha$, and IFN- $\gamma$ in severe asthma (12), or an association of IL-33 and other inflammatory mediators such as IL-5, IL-8, IL-13, and IgE with spontaneous pulmonary inflammation in mice (14). Finally, the increased mRNA expression of IL-33 in human and murine lung epithelial cells after infection with different IAV strains further strengthens the relevance of IL-33 to humans and a possible role of IL-33 during infection with influenza virus.

In conclusion, our findings provide evidence for the profound expression of IL-33 in IAV-infected lung tissue, in correlation with other proinflammatory cytokines. In addition, in vitro experiments demonstrated the important release of IL-33, in accordance with the lysis of infected cells, suggesting a role for this cytokine in virally induced pulmonary infection.

Author Disclosure: None of the authors has a financial relationship with a commercial entity that has an interest in the subject of this manuscript.

Acknowledgments: For the immunohistochemistry analysis, the authors thank the dedicated platforms of IFR140, University of Rennes 1, Rennes, France. The authors are also grateful to Rachel Young for her English-language revision of the manuscript.

\section{References}

1. Schmitz J, Owyang A, Oldham E, Song Y, Murphy E, McClanahan TK, Zurawski G, Moshrefi M, Qin J, Li X, et al. IL-33, an interleukin1-like cytokine that signals via the IL-1 receptor-related protein ST2 and induces T helper Type 2-associated cytokines. Immunity 2005;23: 479-490.

2. Carriere V, Roussel L, Ortega N, Lacorre DA, Americh L, Aguilar L, Bouche G, Girard JP. IL-33, the IL-1-like cytokine ligand for ST2 receptor, is a chromatin-associated nuclear factor in vivo. Proc Natl Acad Sci USA 2007;104:282-287.

3. Kakkar R, Lee RT. The IL-33/ST2 pathway: therapeutic target and novel biomarker. Natl Rev 2008;7:827-840.

4. Komai-Koma M, Xu D, Li Y, McKenzie AN, McInnes IB, Liew FY. IL-33 is a chemoattractant for human Th2 cells. Eur J Immunol 2007; 37:2779-2786.

5. Oboki K, Ohno T, Kajiwara N, Arae K, Morita H, Ishii A, Nambu A, Abe T, Kiyonari H, Matsumoto K, et al. IL-33 is a crucial amplifier of innate rather than acquired immunity. Proc Natl Acad Sci USA 2010.

6. Xu D, Jiang HR, Kewin P, Li Y, Mu R, Fraser AR, Pitman N, KurowskaStolarska M, McKenzie AN, McInnes IB, et al. IL-33 exacerbates antigen-induced arthritis by activating mast cells. Proc Natl Acad Sci USA 2008;105:10913-10918.

7. Pushparaj PN, Tay HK, H'Ng SC, Pitman N, Xu D, McKenzie A, Liew FY, Melendez AJ. The cytokine interleukin-33 mediates anaphylactic shock. Proc Natl Acad Sci USA 2009;106:9773-9778.

8. Mok MY, Huang FP, Ip WK, Lo Y, Wong FY, Chan EY, Lam KF, Xu D. Serum levels of IL-33 and soluble ST2 and their association with disease activity in systemic lupus erythematosus. Rheumatology (Oxford) 2010;49:520-527.

9. Marvie P, Lisbonne M, L'Helgoualc'h A, Rauch M, Turlin B, Preisser L, Bourd-Boittin K, Theret N, Gascan H, Piquet-Pellorce C, et al. Interleukin-33 overexpression is associated with liver fibrosis in mice and humans. J Cell Mol Med 2010;14:1726-1739.

10. Nishida A, Andoh A, Imaeda H, Inatomi O, Shiomi H, Fujiyama Y. Expression of interleukin 1-like cytokine interleukin 33 and its receptor complex (ST2L and IL1RACP) in human pancreatic myofibroblasts. Gut 2009;59:531-541.

11. Moussion C, Ortega N, Girard JP. The IL-1-like cytokine IL-33 is constitutively expressed in the nucleus of endothelial cells and epithelial cells in vivo: a novel "alarmin"? PLOS ONE 2008;3:e3331.

12. Prefontaine D, Lajoie-Kadoch S, Foley S, Audusseau S, Olivenstein R Halayko AJ, Lemiere C, Martin JG, Hamid Q. Increased expression of IL-33 in severe asthma: evidence of expression by airway smooth muscle cells. J Immunol 2009;183:5094-5103.

13. Hayakawa H, Hayakawa M, Kume A, Tominaga S. Soluble ST2 blocks interleukin-33 signaling in allergic airway inflammation. $\mathrm{J}$ Biol Chem 2007;282:26369-26380.

14. Zhiguang X, Wei C, Steven R, Wei D, Wei Z, Rong M, Zhanguo L, Lianfeng Z. Over-expression of IL-33 leads to spontaneous pulmonary inflammation in mIL-33 transgenic mice. Immunol Lett 2010;131: 159-165.

15. Walzl G, Matthews S, Kendall S, Gutierrez-Ramos JC, Coyle AJ, Openshaw PJ, Hussell T. Inhibition of ST1/ST2 during respiratory 
syncytial virus infection prevents T helper cell Type 2 (Th2)- but not Th1-driven immunopathology. J Exp Med 2001;193:785-792.

16. Dawood FS, Jain S, Finelli L, Shaw MW, Lindstrom S, Garten RJ, Gubareva LV, Xu X, Bridges CB, Uyeki TM. Emergence of a novel swine-origin influenza A (H1N1) virus in humans. N Engl J Med 2009; 360:2605-2615

17. Peiris JS, Cheung CY, Leung CY, Nicholls JM. Innate immune responses to influenza A H5N1: friend or foe? Trends Immunol 2009;30:574584.

18. Chevalier C, Al Bazzal A, Vidic J, Fevrier V, Bourdieu C, Bouguyon E, Le Goffic R, Vautherot JF, Bernard J, Moudjou M, et al. PB1-F2 influenza A virus protein adopts a beta-sheet conformation and forms amyloid fibers in membrane environments. J Biol Chem 2010; 285:13233-13243.

19. Le Goffic R, Balloy V, Lagranderie M, Alexopoulou L, Escriou N, Flavell R, Chignard M, Si-Tahar M. Detrimental contribution of the Toll-like receptor (TLR) 3 to influenza A virus-induced acute pneumonia. PLoS Pathog 2006;2:e53.
20. Wikenheiser KA, Vorbroker DK, Rice WR, Clark JC, Bachurski CJ, Oie HK, Whitsett JA. Production of immortalized distal respiratory epithelial cell lines from surfactant protein C/simian virus 40 large tumor antigen transgenic mice. Proc Natl Acad Sci USA 1993;90: 11029-11033.

21. Masamune A, Watanabe T, Kikuta K, Satoh K, Kanno A, Shimosegawa $\mathrm{T}$. Nuclear expression of interleukin-33 in pancreatic stellate cells. Am J Physiol 2010;299:G821-G832.

22. Zhao $\mathrm{W}, \mathrm{Hu} \mathrm{Z}$. The enigmatic processing and secretion of interleukin33. Cell Mol Immunol 2010;7:260-262.

23. Hou CC, Zhao HJ, Cai SX, Li WJ, Tong WC, Liu LY. Respiratory syncytial virus increases the expression and release of high mobility group box-1 protein in the lung tissue of mice [in Chinese]. Nan Fang Yi Ke Da Xue Xue Bao [J South Med Univ] 2010;30:700-703.

24. Kayamuro H, Yoshioka Y, Abe Y, Arita S, Katayama K, Nomura T, Yoshikawa $\mathrm{T}$, Kubota-Koketsu $\mathrm{R}$, Ikuta $\mathrm{K}$, Okamoto $\mathrm{S}$, et al. Interleukin-1 family cytokines as mucosal vaccine adjuvants for induction of protective immunity against influenza virus. J Virol 2010. 